\title{
Dangers in the beautiful ocean; a case report of anaphylaxis due to a jellyfish sting
}

\author{
Ruben $\mathbf{J}^{1}$, Sasanka PLL ${ }^{2}$, Aloka WPP ${ }^{2}$, Sirisena DC $^{3}$, De Silva MAH ${ }^{2}$, Yahathugoda TC ${ }^{1}$ \\ ${ }^{\prime}$ Department of Parasitology, Faculty of Medicine, University of Ruhuna, Galle, Sri Lanka. \\ ${ }^{2}$ Teaching Hospital Karapitiya, Galle, Sri Lanka. ${ }^{3}$ Community Fort Foundation, Fort, Galle, Sri Lanka.
}

Correspondence: Dr. Janaka Ruben

e-mail: janakaruben@med.ruh.ac.lk

D https://orcid.org/0000-0001-6391-8255

\section{Background}

Increasing number of jellyfish attacks have been reported in Sri Lankan media recently. Injuries due to jellyfish sting were reported island wide in June, 2015 and it created a panic among public. Many were hospitalized due to jellyfish attacks occurred in Galle Face, Mount Lavinia, and Ambalangoda beaches. Subsequently it extended to Polhena and Tangalle beaches resulting in more jellyfish envenomation and hospitalizations (1). A fisherman from Jaffna district has died after being stung by a Jellyfish in October, 2017, even before hospitalization. Leaders of the Jaffna fishermen's societies have declared that 5 to 10 people die annually in north of the island due to jellyfish envenomation (2).

Ongoing research in the Department of Parasitology has revealed several episodes of jellyfish attacks near Galle coast. Case presented here had being stung by a jellyfish near Galle Fort. There were six more injured on the same day at Galle Fort, but none were admitted to Teaching Hospital Karapitiya (THK) and were probably treated at OPD level in private hospitals.

Marine envenomation is not well reported in Sri Lanka due to various reasons. Two third of the costal margin of Sri Lanka was affected by the 30 years long civil war and information from these areas didn't reach us. Some cases were reported as drowning instead of marine envenomation. It is essential that the medical officers, especially those who are working in coastal hospitals, should possess adequate knowledge in clinical presentation, firstaid, management of stings and its complications and prevention of marine envenomation. Therefore, a case of complicated jellyfish sting is described here.

\section{Case report}

We present here a 10 year-old boy who developed an anaphylaxis due to a jellyfish sting. The injury occurred while he was bathing in the sea near Galle Fort around 4.00 p.m. on $18^{\text {th }}$ July 2018 . The boy experienced a sudden onset localized pain over the medial surface of the right forearm and felt like a bite from sharp teeth. Simultaneously he noticed a bluish coloured substance in the water close to the place where the pain arose. "In a flicker the substance moved away" the boy said. Immediately after, he experienced tightening of the chest and difficulty in breathing, abdominal cramps and pain in both arm pits. He was taken to a private hospital situated 10 minutes away from the incident. On examination around 4.30 p.m., medical officer recorded weak and rapid pulse (112 beats per minute), low blood pressure $(80 / 60 \mathrm{mmHg})$, respiratory rate of 24 breaths per minute and rhonchi in both lungs. An intramuscular adrenaline (0.3 mg, $1: 1000)$; parenteral chlorpheniramine and hydrocortisone were administered. Child's blood pressure increased to $120 / 90 \mathrm{mmHg}$ following the treatment. However, the medical officer has observed that the patient's abdominal pain was persistent and the respiratory and heart rates were not improved. Therefore the patient was transferred to the Emergency and Trauma Care Centre (ETC) at the Teaching Hospital Karapitiya (THK) around 5.00 p.m.

On arrival at the ETC, child had mild dyspnoea and strider. His pulse rate was $112 \mathrm{bpm}$, blood pressure was $118 / 70 \mathrm{mmHg}$, respiratory rate was $20 \mathrm{bpm}$, oxygen saturation recorded $100 \%$ in room air and GCS was $15 / 15$. He had four blackish skin lesions 
resembling burn marks over contact sites with mild swelling (Figure 1). Whole blood clotting time was less than 20 minutes. He was kept under observation in the ETC and transferred to a ward around 7.15 p.m. since all vital parameters were within normal range.

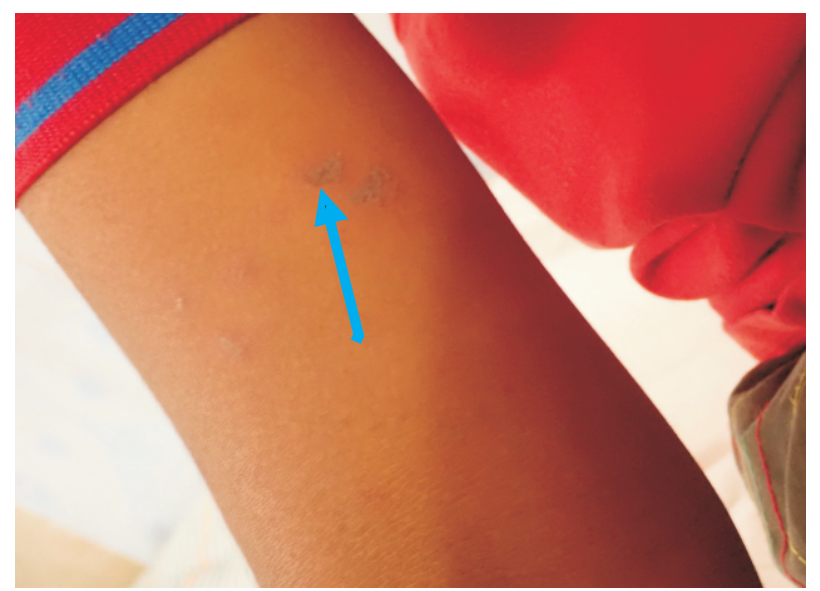

Figure 1: Burn like skin lesions over the contact sites

His C-reactive protein was less than $2 \mathrm{mg} / \mathrm{L}$. Local inflammation was more marked with red tender swelling around the lesions. He was treated with IV hydrocortisone and oral cloxacillin, oral metronidazole (200 $\mathrm{mg}-8$ hourly) and oral chlorpheniramine. He was kept in the ward till third day due to marginally elevated blood pressure compared to his age and discharged on oral antihistamine, oral and topical antibiotics. The patient was followed-up for two weeks. Patient did not complain any significant event afterwards and the skin lesions turned into light patches at two weeks (Figure 2).

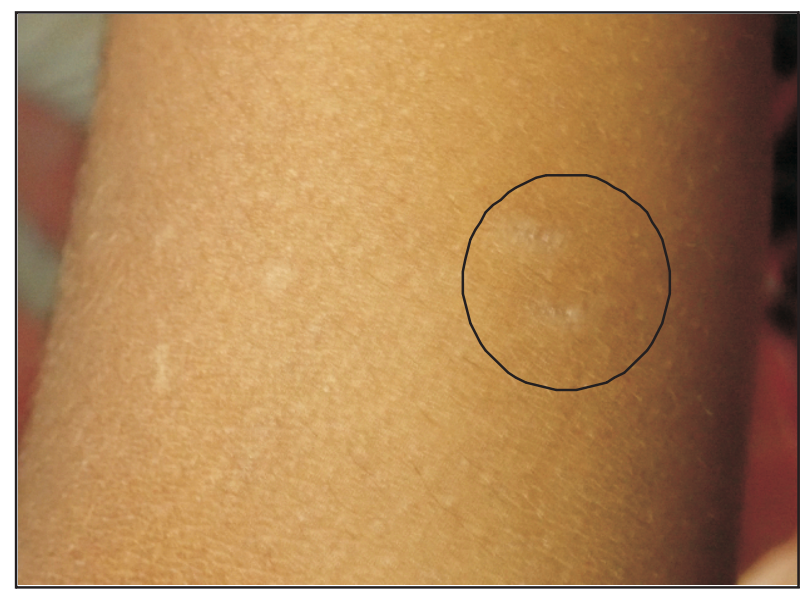

Figure 2: Healed skin lesions over the contact site

\section{Discussion}

The patient explained his pain similar to a bite with sharp teeth. However, there were no bite marks or bleeding but there were non-specific dark bluish skin spots resembling burn marks. Therefore, a bite of a sea creature is unlikely. Such burn marks can be caused by a box jellyfish. Unfortunately our 3 days search in the reef where incidence happened ended up by finding only Portuguese man-o'-war species. The species we found in the vicinity known to cause linear macula popular eruptions which is different to the current presentation $(3,4)$. According to the patient's description ("blue thing moving away in a flicker"), and the lesions resembling burn marks (not eruptions), the causative agent is more likely a box-jellyfish rather than Portuguese man-o'-war (4).

Chiropsalmus buitendijki (Box-jelly fish) comes under the subphylum Medusozoa belonging to phylum Clindaria. There are more than 10,000 aquatic species in phylum Clindaria, of which around 100 are known to cause injury to humans. Jellyfish stings are not much documented in health sector of Sri Lanka. However some venomous species like Cyanea purpurea (Blue jellyfish / Lion's mane jellyfish), Chrysaora quinquecirra (sea nettle, Compass jellyfish) and Physalia spescies (Protuguese man-o'-war / blue bottle) other than box-jellyfish are well identified and documented (4). There are three groups of jellyfish causing (i) fatal stings (ii) severe envenomation with systemic effects and (iii) nuisance stings (6). Extensive work carried out by Fernando, 2001 found that all identified in Sri Lankan western coast happened to be either (ii) or (iii) mentioned above (4).

Injuries are caused by the venom containing nematocysts located in tentacles (Figure 3). Envenomation could occur through just touching non-active nematocysts or active nematocysts (barbed thread has come out) or barbed threads passing through skin tissue $(5 ; 7)$. The toxin delivered through barbed thread could affect muscle, nerve and other tissues (7).

Jellyfish can have neurotoxins, cardiotoxins, cytotoxins, haematotoxins and dermatonecrotic toxins (7). Therefore jellyfish can cause a range of clinical symptoms in case of envenomation. Jellyfish 
envenomation is usually expressed as the immediate appearance of pain, burning sensation and a redness with a subsequent papulo-vesicular eruption or blackish darken skin lesions resembling burn marks of the involved skin. Symptoms are due to immediate allergic, acute toxic and persistent inflammatory responses $(4,8,9)$. Physalia sp. can cause erythema in a form of beaded streaks, bearable pain and distress. These symptoms can resolve within 24 hours. Cyanea sp. can cause rapid development of painful erythema and weal and sometimes rows of erythematous papules. Mildly pruritic weal will take several days to subside whereas severe pain and erythema may disappear within few hours. Box-jellyfish sting can cause pain which develop slowly and less severe compared that of Physalia and Cyanea species. However, sudden appearance of erythematous weal is seen and stained lesions can last for several days with box-jellyfish sting. Chrysaora sp. can cause sudden onset most painful sting. Erythematous weal caused by Chrysaora sp. reaches its maximum size very quickly and disappears within 30 minutes (4).

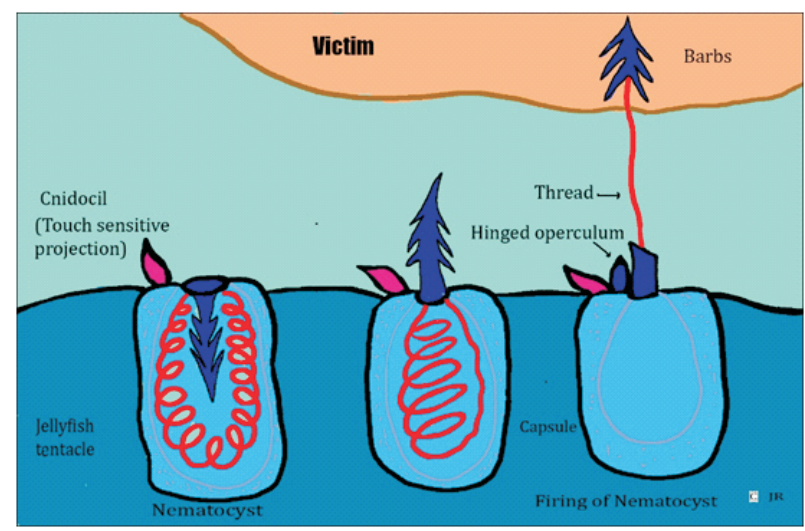

Figure 3: The mechanism of nematocyst firing

When the list of symptoms and the time taken to appear symptoms are considered, it seems that the injury of the present case was caused by a boxjellyfish. The principal author has located and identified box-jellyfish in the same vicinity in a different occasion during the south-east monsoon period (May to September). Children are more prone to get anaphylaxis following jellyfish sting due to many reasons. Scientists have noticed that the toxin's effect is much stronger in children than that of older victims. Thus a child could die because of severe toxicity (7).
Although in Sri Lanka envenomation following jellyfish stings is mostly self-limited and mild, serious allergic reactions including anaphylaxis and fatal stings have been reported worldwide $(4,5,10$,). Several fatal cases, however, have been reported by fishermen in Jaffna peninsula (2). Thus having basic knowledge of jellyfish sting management is important for medical officers working in coastal areas. Many solutions are used to wash the sting site as a first-aid step. Commonly used ones are hot water $\left(60{ }^{\circ} \mathrm{C}\right)$, vinegar, acetic acid, coca cola and old wine. Urinating on tentacles, use papain, aluminum sulfate, ethanol, bleach, ammonia, kerosene or rubbing sand on the affected area also advocated. However some of these solutions like vinegar might precipitate nematocyst firing when the sting is from Chrysaora sp (11). Fresh water in ambient temperature should not be used for rinsing sting sites because it could increase the nematocyst toxin release (7). Therefore, in our settings it is better to advise the victims to use sea water or hot water to rinse the affected site to stop further venom release by nematocysts and rush to a closest medical care facility. Topical application of a local anesthetic gel, an antihistamine cream, or a corticosteroids cream may be helpful to relieve the local symptoms. Persistent severe pain may require strong painkillers like morphine. Muscle cramps may respond to muscle relaxants like diazepam.

All swimmers must be educated with preventive techniques such as wearing tight fitting long cloths covering entire body and use of diving shoes and helmets or swim in a stringer resistant net. Swimming in cleared areas with lifeguards while obeying warning signs is always recommended.

\section{Conclusions and recommendations}

A clinical history of painful stings sustained during sea bathing with dark skin marks resembling to burn marks are highly suggestive of a jellyfish sting. Such patients should not be ignored due to potential danger of life threatening anaphylactic reactions. Medical undergraduate and postgraduate prospectuses should be improved by adding marine envenomation and its managements under toxicology. 


\section{References}

1. Sunday Island, June 23, 2015, e News article URL: http://www.island.lk/index.php?page_cat=articledetails\&page $=$ article-etails \& code_title $=127074$, [Accessed 06.09.2018.]

2. Sunday Times, June 21, 2015, e News article URL: http://www.sundaytimes.lk/150621/news/poisonscentre-docs-throw-the-booklet-at-jellyfish-154356.html. [Accessed 06.09.2018.]

3. Fernando M. Some hazards of diving. Ceylon Medical Journal, 1992; 37: 72-80.

4. Fernando M. Hunting jellyfish. Ceylon Medical Journal, 2001; 46(4): 139-40.

5. Silfen R, Vilan A, Wohl I, Leviav A. Mediterranean jellyfish (Rhopilema nomadica) sting. Burns, 2003; 29(8): 868-70.

6. Fenner PJ. Dangers in the ocean: the traveler and marine envenomation. II. Marine vertebrates. Journal of Travel Medicine, 1998;5(4): 213-216.

7. Mujiono N. Jellyfish sting: an Indonesian case report. Journal Ilmiah Perikanan dan Kelautan, 2010; 2(1): 1-9.
8. Uri S, Marina G, Liubov G. Severe delayed cutaneous reaction due to Mediterranean jellyfish (Rhopilema nomadica) envenomation. Contact Dermatitis. 2005; 52(5): 282-283.

9. Menahem S, Shvartzman P. Recurrent dermatitis from jellyfish envenomation. Canadian Family Physician 1994; 40: 2116-8.

10. Lotan A, Fishman L, Zlotkin E. Toxin compartmentation and delivery in the Cnidaria: the nematocyst's tubule as a multiheaded poisonous arrow. Journal of Experimental Zoology, 1996; 275(6): 444-51.

11. Yoffe B, Baruchin AM. Mediterranean jelly fish (Rhopilema nomadica) sting. Burns, 2004; 30(5): 503-4.

12. Wolfe J. Taking the "Sting" Out of Jellyfish, August, 2011 [Available from: https://www.esdhd.org/PDF/ Reference/jelly-Fish-stings.pdf. 